\title{
Recombinant Tumor Suppressor TSC1 differentially interacts with E.coli DnaK and human HSP70
}

Nalini Natarajan ${ }^{1}$, Althaf Shaik ${ }^{2}$, Vijay Thiruvenkatam ${ }^{1 *}$

1. Discipline of Biological Engineering, Indian Institute of Technology Gandhinagar, Simkheda, Palaj, Gandhinagar, 382355, Gujarat, India

2. Discipline of Chemistry, Indian Institute of Technology Gandhinagar, Simkheda, Palaj, Gandhinagar, 382355, Gujarat, India

‘*' Corresponding Author

Contact information: vijay@iitgn.ac.in, Phone: +91-9925907251

Supplementary information:

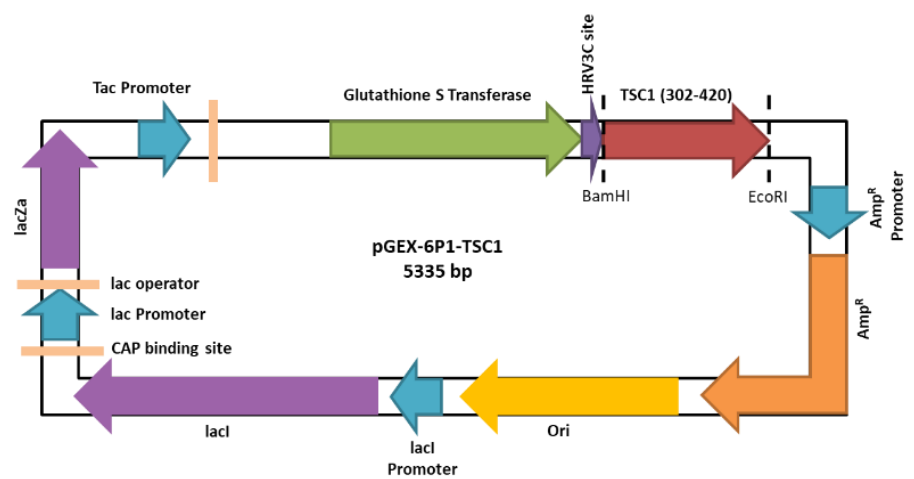

Figure S1: The pGEX-6P1 vector map containing TSC1 gene corresponding to 302-420 residues

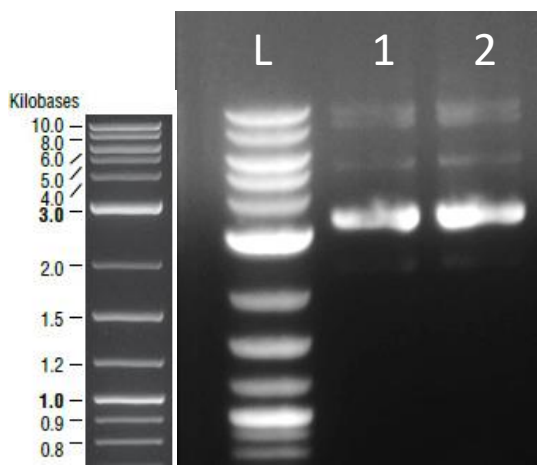

Figure S2. Agarose gel electrophoresis: Agarose gel showing the TSC cloned plasmids. Lane L represents the DNA ladder (1Kb). Lane 1 \& 2 shows pGEX-6P1-TSC1 (TSC1 translates to TSC1 302-420 aа). 


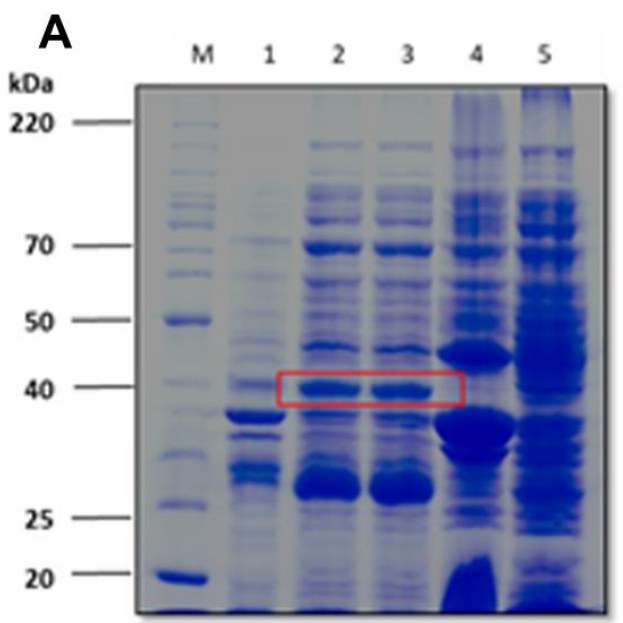

B

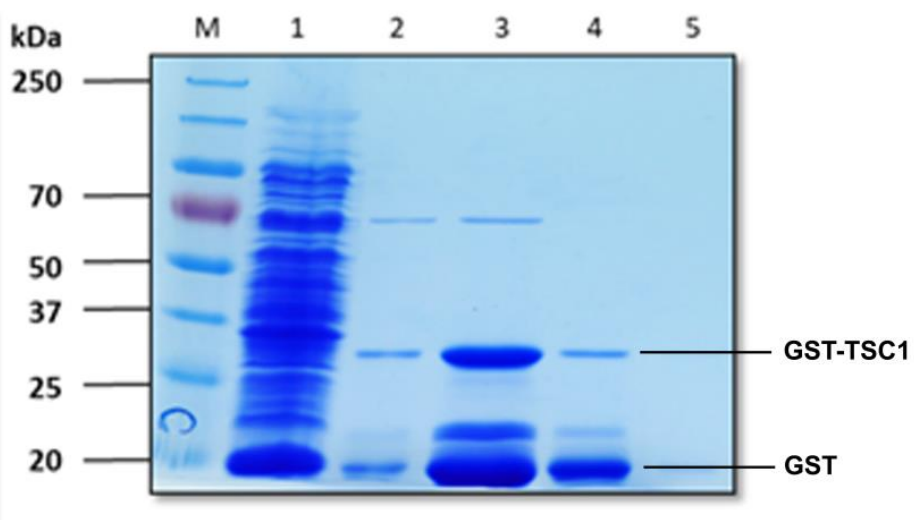

Figure S3: A Expression of GST-TSC1 (302-420 aa) at $18^{\circ} \mathrm{C}$ : Lane 1- Induced with $0.5 \mathrm{mM}$ IPTG insoluble fraction, Lane-2 \& 3- Induced with $0.5 \mathrm{mM}$ IPTG soluble fractions, Lane 4- Uninduced insoluble fraction, Lane 5- Uninduced soluble fraction. Lane M - Molecular weight ladder in $\mathrm{kDa}(220$, $180,120,100,90,80,70,60,50,40,30,25,20)$. The bands representing soluble GST-TSC1 (302-420 aa) and GST-TSC2 (1-418 aa) at the best expressed conditions are enclosed in a red box. B SDS-PAGE analysis after GST affinity purification of TSC1 (302-420 aa). Lane 1- Unbound fraction, Lane 2Elution fraction 1 showing prominent GST-TSC1 (302-420 aa) at $40 \mathrm{kDa}$ and GST at $26 \mathrm{kDa}$. Lane 3Elution fraction 2. Lane 4- Elution fraction 3. Lane 5- Elution fraction 4. Lane M- Molecular weight ladder in $\mathrm{kDa}(170,130,95,72,55,43,34,26)$

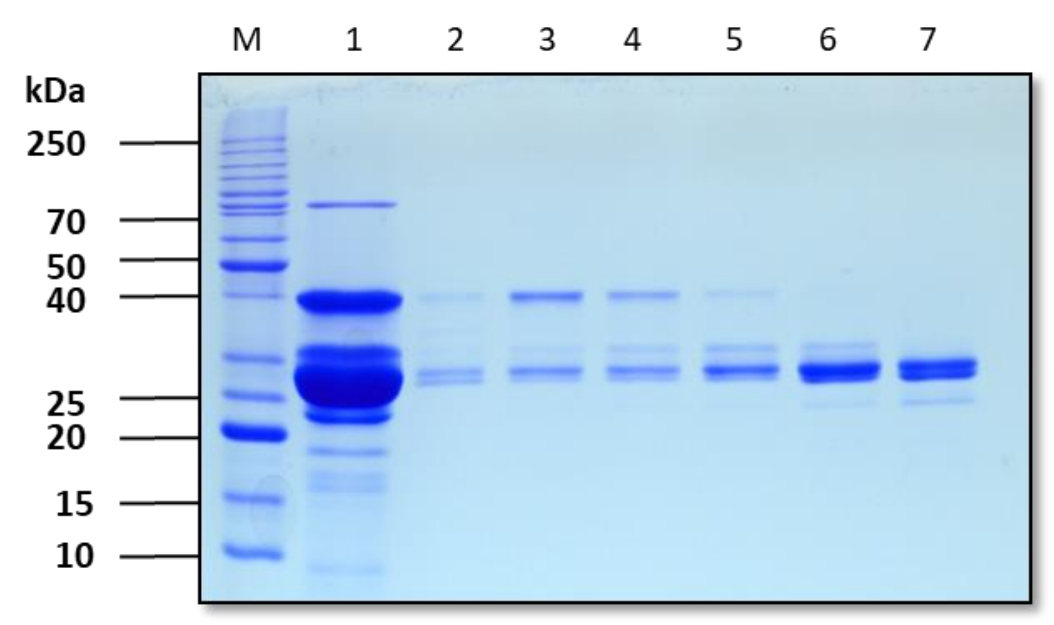

Figure S4. Size-exclusion chromatography: SDS-PAGE after Glutathione affinity chromatography followed by size-exclusion chromatography. The GST-TSC1 and GST proteins could not be solved in size-exclusion chromatography. This could be due to narrow size range as well as possible interaction between GST. Lane 1: Sample before size-exclusion. Lane 2-7: Fractions out of the size-exclusion column.

For purification of GST-tagged TSC1, the supernatant containing the soluble protein was added to the PBS equilibrated GST resin and was kept for binding at $4^{\circ} \mathrm{C}$ for $8 \mathrm{hrs}$. The matrix 
was then packed in a gravity flow column (Bio-Rad) and washed with PBS to remove the unbound. The GST fused proteins were eluted by using elution buffer ( $50 \mathrm{mM}$ Tris base, 10 $\mathrm{mM}$ reduced glutathione, $\mathrm{pH} 8.0$ ) in $5 \mathrm{ml}$ fractions. The presence of the required band size is checked by performing SDS-PAGE. Followed by the GST affinity chromatography, size exclusion was performed. We have observed non-specific protease activity that cleaved between GST and TSC1, which resulted in free GST and loss of TSC1. Also, the size exclusion chromatography resulted in co-elution of GST impurity along with the fusion protein (Figure S4), hence we had to use a different methodology to obtain pure TSC1 (Figure S2).

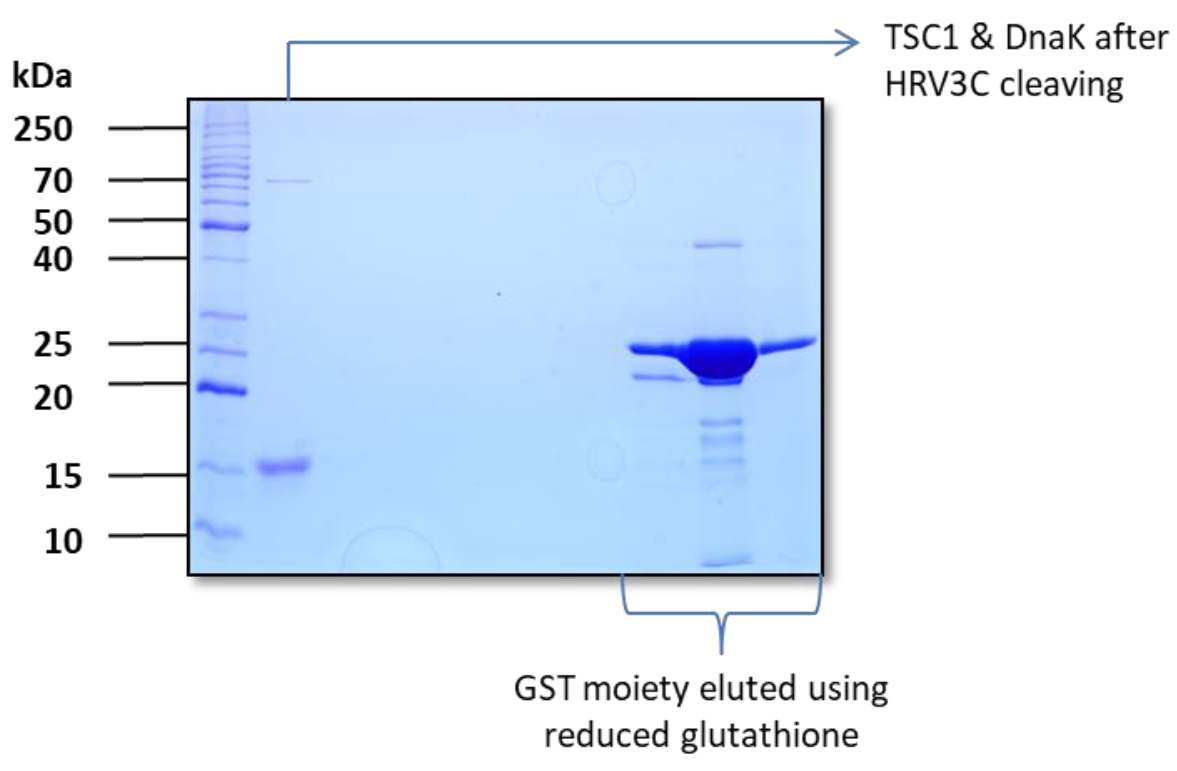

Figure S5. On-Column cleavage: SDS-PAGE after on-column cleaving. After incubation with precission protease (HRV3C), TSC1 and DnaK came in the unbound fraction. The resultant protein bound to the column when eluted with glutathione contained majorly GST and traces of uncleaved GST-TSC1.

\title{
MATRIX MASCOT Search Results
}

\author{
Protein View: TSC1_HUMAN \\ Hamartin OS=Homo sapiens $\mathrm{GN}=\mathrm{TSC} 1 \mathrm{PE}=1 \mathrm{SV}=2$ \\ Database: SwissProt \\ Score: 78 \\ Nominal mass $\left(\mathrm{M}_{\mathrm{r}}\right)$ : 129685 \\ Calculated pI: $\quad 6.02$ \\ Taxonomy: Homo sapiens
}

Sequence similarity is available as an NCBI BLAST search of ISC1 HUMAN against nr. 
Figure S6. LC-ESI MS/MS of TSC1: The SDS-gel sample stained with Coomassie brilliant blue was excised and sent of the mass analysis (IISc Bangalore, Karnataka, India). The raw data obtained was uploaded in the MASCOT server (Matrix Science Inc., MA, USA). The peptide mass fingerprinting showed the presence of TSC1.

\begin{tabular}{|c|c|c|c|c|c|c|c|c|c|c|c|}
\hline \multicolumn{2}{|l|}{ Protein Groups } & \multicolumn{2}{|c|}{ Peptide Groups } & PSMs & MS/MS Spectrum Info & Input Files $\%$ & \multicolumn{2}{|c|}{ Specialized Traces } & & \multirow[b]{2}{*}{ Coverage [\%] } & \multirow[b]{2}{*}{ II Peptide: } \\
\hline Protein FD $\boxminus$ & Master & Accession & \multicolumn{4}{|c|}{ Description } & Exp. Q-valu & SUM PEP & Score - & & \\
\hline High & $\checkmark$ & A7ZHA4 & \multicolumn{4}{|c|}{ Chaperone protein DnaK OS=Escherichia coli O139.H28 (si | } & 0.000 & & 89.759 & $51 \%$ & 27 \\
\hline High & 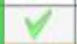 & POAG69 & \multicolumn{4}{|c|}{30 S ribosomal protein \$1 0S-Escherichia coli 0157:H7 GN } & 0.000 & & 3.067 & $10 \%$ & 4 \\
\hline High & V & $\mathrm{P} 09373$ & \multicolumn{4}{|c|}{ Formate acetyltransferase 1 OS=Escherichia coli (strain K1: } & 0.000 & & 2.653 & $4 \%$ & 3 \\
\hline High & $\vee$ & P63285 & \multicolumn{4}{|c|}{ Chaperone protein ClpB OS-Escherichis coli 0157. H7 GN. } & 0.000 & & 2.230 & $2 \%$ & 1 \\
\hline High & $\forall$ & P00490 & \multicolumn{4}{|c|}{ Maltodextrin phosphorylase $\mathrm{OS}=$ Escherichia coli (strain $\mathrm{K} 12$} & 0.000 & & 2.136 & $3 \%$ & 2 \\
\hline High & V & A1A809 & \multicolumn{4}{|c|}{ Chaperone protein HtpG OS-Escherichia coli O1:K1 / APEC } & 0.000 & & 1.784 & $6 \%$ & 3 \\
\hline High & $\checkmark$ & POAC43 & \multicolumn{4}{|c|}{ Succinate dehydrogenase flavoprotein subunit OS=Escheri } & 0.000 & & 1.504 & $2 \%$ & 1 \\
\hline High & V & A7ZLA9 & \multicolumn{4}{|c|}{ Exoribonudease 2 OS-Escherichia coli 0139:H28 (strain E } & 0.000 & & 1.112 & $2 \%$ & 1 \\
\hline High & $\forall$ & P27298 & \multicolumn{4}{|c|}{ Oligopeptidase A OS=Escherichia coli (strain K12) GN=prIC } & 0.000 & & 1.039 & $1 \%$ & 1 \\
\hline
\end{tabular}

Figure S7. LC-ESI MS/MS of DnaK: Peptide mass fingerprinting of DnaK. The SDS-gel sample stained with Coomassie brilliant blue was excised and sent of the mass analysis (IIT Bombay, India). The raw data obtained was uploaded in the MASCOT server (Matrix Science Inc., MA, USA). The peptide mass fingerprinting showed the presence of TSC1.

CLUSTAL O(1.2.4) multiple sequence alignment

2

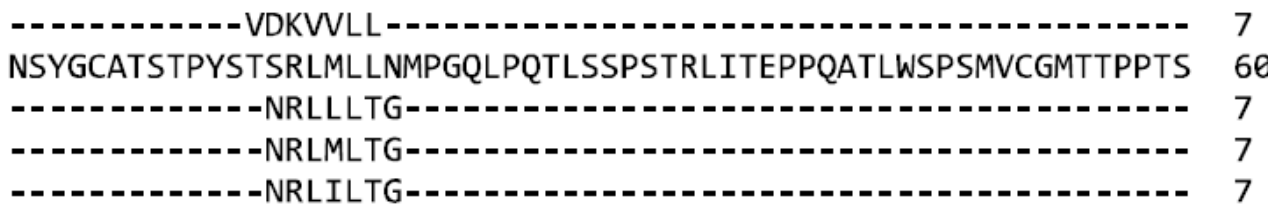

Figure S8. Multiple sequence alignment: N-terminal 1-60 residues of TSC1 is aligned with the peptide sequences that are known to interact with DnaK. 


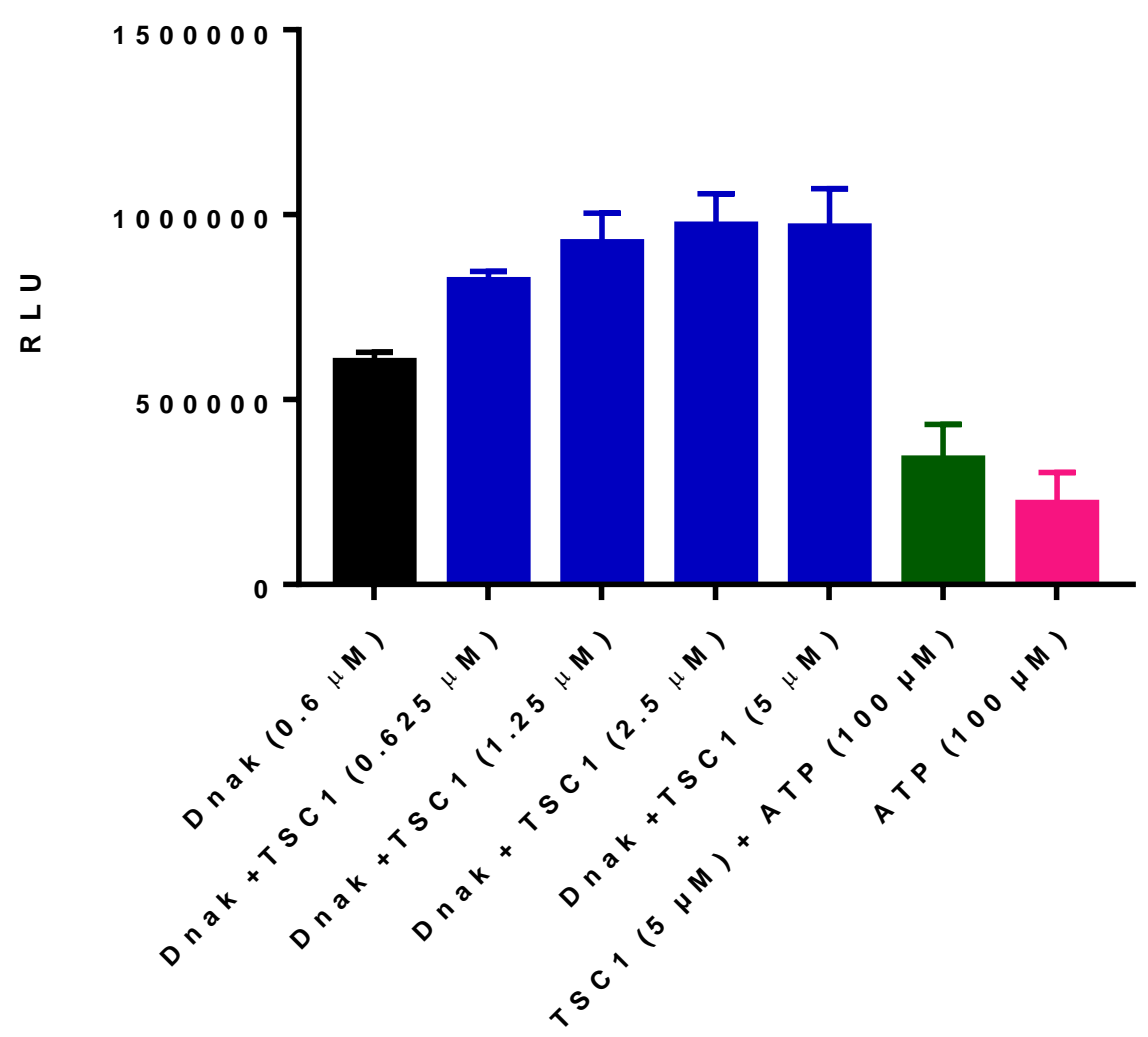

Figure S9. DnaK ATPase activity: ATPase activity of DnaK measured as luminescence through ADP$\mathrm{Glo}^{\mathrm{TM}}$ assay. The black bar denotes the activity of $0.6 \mu \mathrm{M}$ of DnaK. The increasing trend in the blue bar denotes the increasing concentration of TSC1 $(0.625 \mu \mathrm{M}, 1.25 \mu \mathrm{M}, 2.5 \mu \mathrm{M}, 5 \mu \mathrm{M})$ added to 0.6 $\mu \mathrm{M}$ of DnaK. The green and pink bars are TSC1 and ATP controls for the experiment. RLU- Relative light unit. 